\title{
Endometrial Evaluation by Transvaginal Ultrasonography Saline Infusion Sonohysterography, Hysteroscopy versus Endometrial Office Bipsy in Perimenopausal Bleeding: A Comparative Study
}

\author{
Mohamed Kamal1*, Almandouh H. Bosilah² \\ ${ }^{1}$ Consultant Fetal Medicine, Fetal Medicine Unit, Department of Obstetrics and Gynecology, Ain Shams University, Cairo, Egypt \\ ${ }^{2}$ Lecturer in Department of Obstetrics and Gynecology, Fayoum University, Fayoum, Egypt \\ Email: *mohamedkamal.eg@outlook.com
}

How to cite this paper: Kamal, M. and Bosilah, A.H. (2020) Endometrial Evaluation by Transvaginal Ultrasonography Saline Infusion Sonohysterography, Hysteroscopy versus Endometrial Office Bipsy in Perimenopausal Bleeding: A Comparative Study. Open Journal of Obstetrics and Gynecology, 10, 1093-1106.

https://doi.org/10.4236/ojog.2020.1080103

Received: July 7, 2020

Accepted: August 21, 2020

Published: August 24, 2020

Copyright $\odot 2020$ by author(s) and Scientific Research Publishing Inc. This work is licensed under the Creative Commons Attribution International License (CC BY 4.0).

http://creativecommons.org/licenses/by/4.0/ Open Access

\begin{abstract}
Postmenopausal bleeding is a very common and alarming symptom accounting for 5 percent of all gynecological outpatients' clinic attendances. This cross-section study was conducted in the Department of Obstetrics and Gynecology at University Hospitals and included 176 patients with perimenopausal bleeding who attended a patient clinic during the period from January 2017 to May 2019. All patients were subjected to transvaginal ultrasound, saline sonohysterography and out-patient endometrial sampling using pipelle; all the results were compared to histopathology obtained by hysteroscopic guided biopsy and/or hysterectomy. Result: In comparing the validity of TVS and SHG we found highly significant decrease in malignancy assessment in the TVS method compared to hysteroscopic-guided biopsy. According to the validity of pipelle in detection of malignant lesion, it was found to detect 80 cases out of 88 cases of malignancy with sensitivity $90.9 \%$, specificity $100 \%$ and 95.45\% accuracy with respect to histopathology by hysteroscopy guided biopsy and/or hysterectomy. Combination tests were done between SHG and pipelle and we found that the validity of combination tests in detecting malignancy improves the sensitivity and specificity in detection of malignancy sensitivity = $100 \%$ and specificity $=100 \%$ and this was found to be as an effective method in detection of malignancy. Conclusion: When SHG combined to outpatient endometrial sampling "pipelle", the results were the same as hysteroscopy.
\end{abstract}

\section{Keywords}

Perimenopausal Bleeding, Hysteroscopy, Endometrial Office Biopsy 


\section{Introduction}

Postmenopausal bleeding (PMB) is characterized as bleeding from the genital tract after at least one year of persistent amenorrhoea in women of menopause or acyclic vaginal bleeding in postmenopausal women with HRT [1]. Postmenopausal bleeding is quite common and worrisome symptom accounting for $5 \%$ of all gynecological outpatients' department attendances [2] [3].

Trans-vaginal ultrasonography (TVS) has been investigated as a non-invasive method of endometrium visualization [4].

Saline contrast sonohysterography (SHG) is a technique in which the endometrial cavity is distended with saline through ultrasonic inspection, which causes the endometrial membrane to be inspected on a single sheet and helps the sonographer to distinguish the focal length from the dispersed endometrial pathological conditions [5].

Over the years, hysteroscopy with direct biopsy has assumed the gold standard investigation for postmenopausal bleeding because it is an accurate method for the diagnosis and treatment of endometrial abnormalities, but its availability, its high cost and the need for general anesthesia sometimes prevent its use as a primary diagnostic procedure in postmenopausal bleeding patients [6]. Gynecologists use endometrial suction biopsy in people with postmenopausal bleeding or whenever endometrial cancer has to be removed [7]. It is a less costly, simple technique for outpatients. Many questions about their precision in identifying severe endometrial disease have been raised recently.

\section{Aim of the Study}

The aim of this is to test the diagnostic performance of transvaginal ultrasound, sonohystrography and office-based endometrial sampling for evaluating the endometrium in women with postmenopausal bleeding as compared to histopathology of hysteroscopic guided biopsy, and/or hysterectomy.

\section{Patients and Methods}

This cross-sectional study was carried out in the department of obstetrics and gynecology at University Hospitals included 176 patients with postmenopausal bleeding attending outpatient clinic during the period from January 2017 to May 2019. Informed consent was obtained from all patients after thorough explanation of all the procedures.

1) Inclusion criteria:

- Patients with post-menopausal bleeding.

\section{2) Exclusion criteria:}

- Patients with abnormal coagulation profile.

- Thyroid gland disorders.

○ Liver diseases.

- Patients presenting with symptoms suggestive of pelvic or lower genital tract infection. 
- Neglected IUD or pessary.

- Any cervical abnormality.

- Cardiac patients.

Women included in the study were subjected to the following:

1) History and test 2) Inquiries:-Full blood count (CBC).

- Examinations of liver and kidney activity, if indicated

- Coagulation profiles.

- Blood glucose level.

- ECG.

Preparation:

1) Trans-vaginal ultrasonography (TVS):

The instrument used is an Ultrasound Unit of General Electric Logic P5 (General Electric, Wauekesha, Wisc., USA) with a curved 5-MHz endovaginal.

\section{2) Saline sonohysterography: \\ Technique of the examination:}

In lithotomy place, a sterile (non-fenestrated) Cusco vaginal speculum was used to expose the cervix, which was then washed by 10 percent (Betadine) of povidone-iodine. 10 Foley's catheter was used in French and added to the cervix. It was sprayed with sterile saline solution before being injected into the endocervical canal through the outer cervical os. It was sprayed with clean saline solution before being injected into the endocervical canal via the outer cervical os. It stopped ecchogenic air bubbles from being created which would have blurred the endometrium. The catheter was introduced into the cervical canal beyond the level of the internal os, its balloon was filled with saline and the catheter was pulled gently.

Next, as the same of TVS the covered tansvaginal probe was inserted into the vagina, and continuous scanning was performed during instillation of fluid. Saline at room temp. $(28-30 \mathrm{c})$ was used and injected into the catheter by syringe 50 millimeter and various volumes of saline solution were used depending on how much was retained within the uterine cavity.

Suspicious mass or suspicious endometrium criteria in TVUS and SHG:

1) Size of the mass

2) Irregular surface

3) Irregular endometrial myometrial junction

4) Non-uniform echogenicity

5) Multiple and multifocal vascular pattern by color Doppler

6) Moderate or high color score

Outpatient endometrial sampling (pipelle):

Attach the speculum. When the uterine fundus was hit, the plunger was completely pushed back to establish optimum downward pressure. The sampler was rotated to scrape the entire uterine cavity using reverse and forward motions inside the uterine depth marks of 30 - 40 s, gathering the endometrial tissue in the lumen. The sampler is extracted and the contents are ejected in a container. 


\section{Hysteroscopy:}

\section{Technique of the examination:}

The uterine cavity was systematically investigated by rotating the 30 fore-oblique scopes. The whole endometrial cavity was closely inspected, including the detection of tubal ostia, the funds and the anterior and posterior parts of uterine wall. Video documentation of the intraoperative findings was done for some cases.

The results of each technique was compared with histopathology obtained from the hysteroscopy guided biopsy, and/or hysterectomy specimen.

\section{Statistical Analysis:}

Data were provided and appropriate analysis was performed according to the type of data (parametric and non-parametric) collected for each element. P-values less than 0.05 (5 percent) were considered to be statistically significant.

\section{Results}

This study included 176 patients with postmenopausal bleeding attending outpatient clinic during the period from January 2017 to May 2019. Table 1 shows that; the mean age of all patients was $(60.8 \pm 5.1)$ years, mean menopausal age was $(51.6 \pm 2.7)$ years, mean parity was $(2 \pm 1)$, and mean gravida was $(1 \pm 1)$. Table 2 shows the diagnostic accuracy of each of the diagnostic tools which show that SHG is more superior than TVS in the diagnosis of different endometrial pathologies. By using ROC-curve analysis, endometrial thickness at TVS method at a cut-off point of $(>9)$, detected patients with malignancy, with good accuracy, sensitivity $=72.73 \%$ and specificity $=90.91 \%(\mathrm{p}<0.01)$. By using ROC-curve analysis, endometrial thickness at SHG method at a cut-off point of $(>5)$, detected patients with malignancy, with good accuracy, sensitivity = $90.91 \%$ and specificity $=86.37 \%(\mathrm{p}<0.0001)$ as shown in Table 3 . By using ROC-curve analysis, TVS method, detected patients with malignancy, with good accuracy, sensitivity $=63 \%$ and specificity $=100 \%(\mathrm{p}<0.01)$. By using ROC-curve analysis, SHG method, detected patients with malignancy, with excellent accuracy, sensitivity $=91 \%$ and specificity $=100 \%(\mathrm{p}<0.01)$. By using ROC-curve analysis, Pipelle method, detected patients with malignancy, with excellent accuracy (Table 4).

Figure 1: ROC curve of TVS method. By using ROC-curve analysis, endometrial thickness at TVS method at a cut-off point of $(>9)$, detected patients with malignancy, with good accuracy, sensitivity $=72.73 \%$ and specificity $=90.91 \%(\mathrm{p}$ $<0.01)$.

By using ROC-curve analysis, SHG method, detected patients with malignancy, with excellent accuracy, sensitivity $=91 \%$ and specificity $=100 \%(\mathrm{p}<0.01)$ (Figure 2).

By using ROC-curve analysis, Pipelle method, detected patients with malignancy, with excellent accuracy, sensitivity $=90 \%$ and specificity $=100 \%(\mathrm{p}<$ 0.01) (Figure 3). 
Table 1. Basic clinical data among 176 women.

\begin{tabular}{ccc}
\hline Variables & Frequency (\%) \\
\hline Age (years) & $60.8 \pm 5.1^{\star}$ \\
Menopausal age (years) & & $51.6 \pm 2.7$ \\
Parity & & $2 \pm 1$ \\
Gravida & & $1 \pm 1$ \\
Smoking & + ve & $0(0 \%)$ \\
DM & + +ve & $80(45.5 \%)$ \\
HTN & + +ve & $80(45.5 \%)$ \\
\hline
\end{tabular}

${ }^{*}$ Mean \pm SD. HTN: hypertension. DM: diabetes mellitus.

Table 2. Predictive accuracy criteria for endometrial atrophy, hyperplasia, polypoid tumors, myoma and cancer with respect to clinical treatment of TVS, SHG and pipeline.

\begin{tabular}{|c|c|c|c|c|c|}
\hline Pathological diagnosis & Sensitivity & Specificity & PPV & NPV & Accuracy \\
\hline \multicolumn{6}{|l|}{ Hyperplasia } \\
\hline TVS & $100.00 \%$ & $65.00 \%$ & $22.22 \%$ & $100.00 \%$ & $68.18 \%$ \\
\hline SHG & $100.00 \%$ & $100.00 \%$ & $100.00 \%$ & $100.00 \%$ & $100.00 \%$ \\
\hline Pipelle & $100.00 \%$ & $85.00 \%$ & $40.00 \%$ & $100.00 \%$ & $86.36 \%$ \\
\hline \multicolumn{6}{|l|}{ Polyp } \\
\hline TVS & $50.00 \%$ & $95.00 \%$ & $50.00 \%$ & $95.00 \%$ & $90.91 \%$ \\
\hline SHG & $100.00 \%$ & $95.00 \%$ & $66.67 \%$ & $100.00 \%$ & $95.45 \%$ \\
\hline Pipelle & $0.00 \%$ & $100.00 \%$ & $0 \%$ & $90.91 \%$ & $90.91 \%$ \\
\hline \multicolumn{6}{|l|}{ Myoma } \\
\hline TVS & $100.00 \%$ & $100.00 \%$ & $100.00 \%$ & $100.00 \%$ & $100.00 \%$ \\
\hline SHG & $100.00 \%$ & $100.00 \%$ & $100.00 \%$ & $100.00 \%$ & $100.00 \%$ \\
\hline Pipelle & $0.00 \%$ & $100.00 \%$ & $0 \%$ & $90.91 \%$ & $90.91 \%$ \\
\hline \multicolumn{6}{|l|}{ Malignancy } \\
\hline TVS & $63.64 \%$ & $100.00 \%$ & $100.00 \%$ & $73.33 \%$ & $81.82 \%$ \\
\hline SHG & $90.91 \%$ & $100.00 \%$ & $100.00 \%$ & $91.67 \%$ & $95.45 \%$ \\
\hline Pipelle & $90.91 \%$ & $100.00 \%$ & $100.00 \%$ & $91.67 \%$ & $95.45 \%$ \\
\hline \multicolumn{6}{|l|}{ Atrophic } \\
\hline TVS & $100.00 \%$ & $100.00 \%$ & $100.00 \%$ & $100.00 \%$ & $100.00 \%$ \\
\hline SHG & $100.00 \%$ & $100.00 \%$ & $100.00 \%$ & $100.00 \%$ & $100.00 \%$ \\
\hline Pipelle & $60.00 \%$ & $100.00 \%$ & $100.00 \%$ & $89.47 \%$ & $90.91 \%$ \\
\hline
\end{tabular}

Table 3. ROC-curve of endometrial thickness to detect malignancy.

\begin{tabular}{ccccccccc}
\hline Variable & \multirow{2}{*}{ AUC } & \multirow{2}{*}{ SE } & Cut off value $\begin{array}{c}\text { Mean thickness } \\
\text { (benign) }\end{array}$ & $\begin{array}{c}\text { Mean thickness } \\
\text { (malignant) }\end{array}$ & $\begin{array}{c}\text { Sensitivity } \\
\text { (\%) }\end{array}$ & $\begin{array}{c}\text { Specificity } \\
\text { (\%) }\end{array}$ & P value \\
\hline Endometrial thickness at TVS & 0.880 & 0.0507 & $>9$ & $6.2 \pm 3.4$ & $19.2 \pm 10.8$ & 72.73 & 90.91 & $<0.0001^{* *}$ \\
Endometrial thickness at SHG & 0.956 & 0.032 & $>5$ & $3.73 \pm 1.58$ & $16.55 \pm 11.38$ & 90.91 & 86.37 & $<0.0001^{* *}$ \\
\hline
\end{tabular}

ROC (Receiver operating characteristic), AUC $=$ Area under curve, $\mathrm{SE}=$ Standard Error. ${ }^{*} \mathrm{P}$ is highly significant. 
Table 4. ROC-curve of TVS, Pipelle, SHG and (SHG + Pipelle) validity in detecting malignancy.

\begin{tabular}{ccccccccc}
\hline Variable & AUC & SE & Sensitivity (\%) & Specificity (\%) & PPV & NPV & Accuracy & P value \\
\hline TVS validity & 0.818 & 0.0525 & 63.64 & 100 & 100 & 73.33 & 81.82 & $<0.0001^{* *}$ \\
Pipelle validity & 0.955 & 0.0314 & 90.9 & 100 & 100 & 91.67 & 95.45 & $<0.0001^{\star *}$ \\
SHG validity & 0.955 & 0.0314 & 91 & 100 & 100 & 91.67 & 95.45 & $<0.0001^{\star *}$ \\
(SHG + Pipelle) validity & 1.000 & 0 & 100 & 100 & 100 & 100 & 100 & $<0.0001^{* *}$ \\
\hline
\end{tabular}

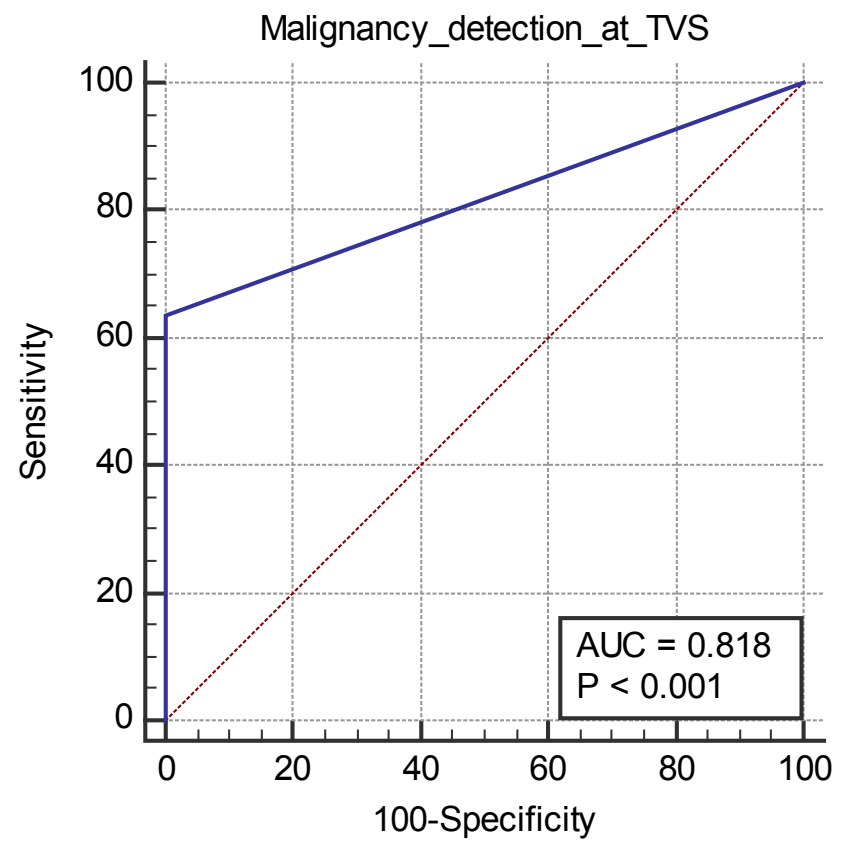

Figure 1. ROC curve of TVS validity.

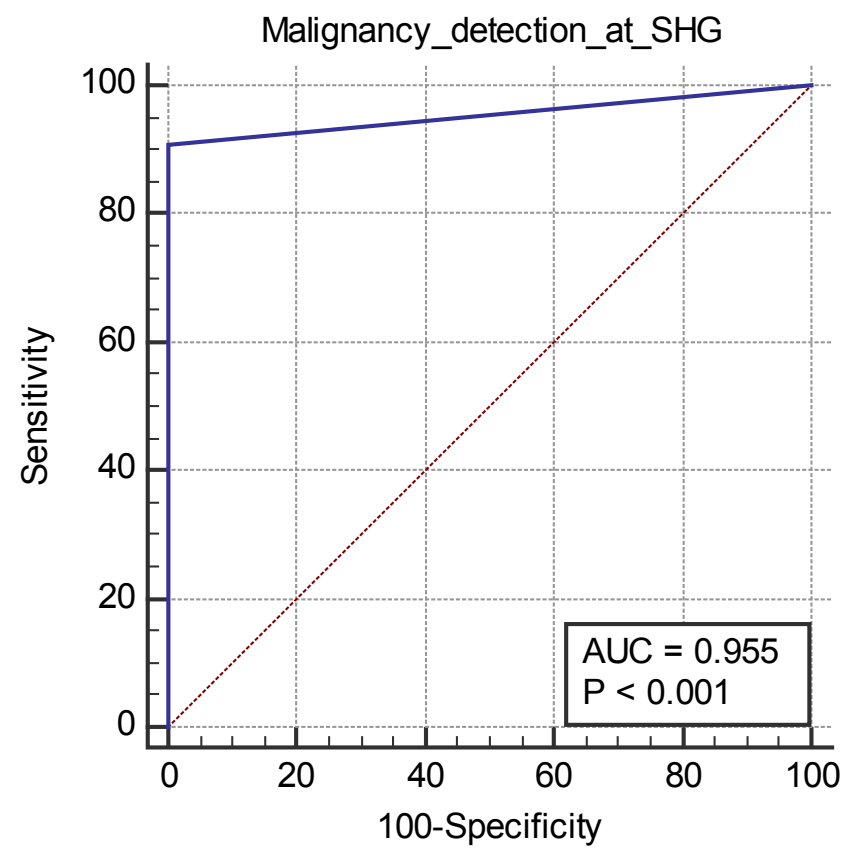

Figure 2. ROC curve of SHG validity. 


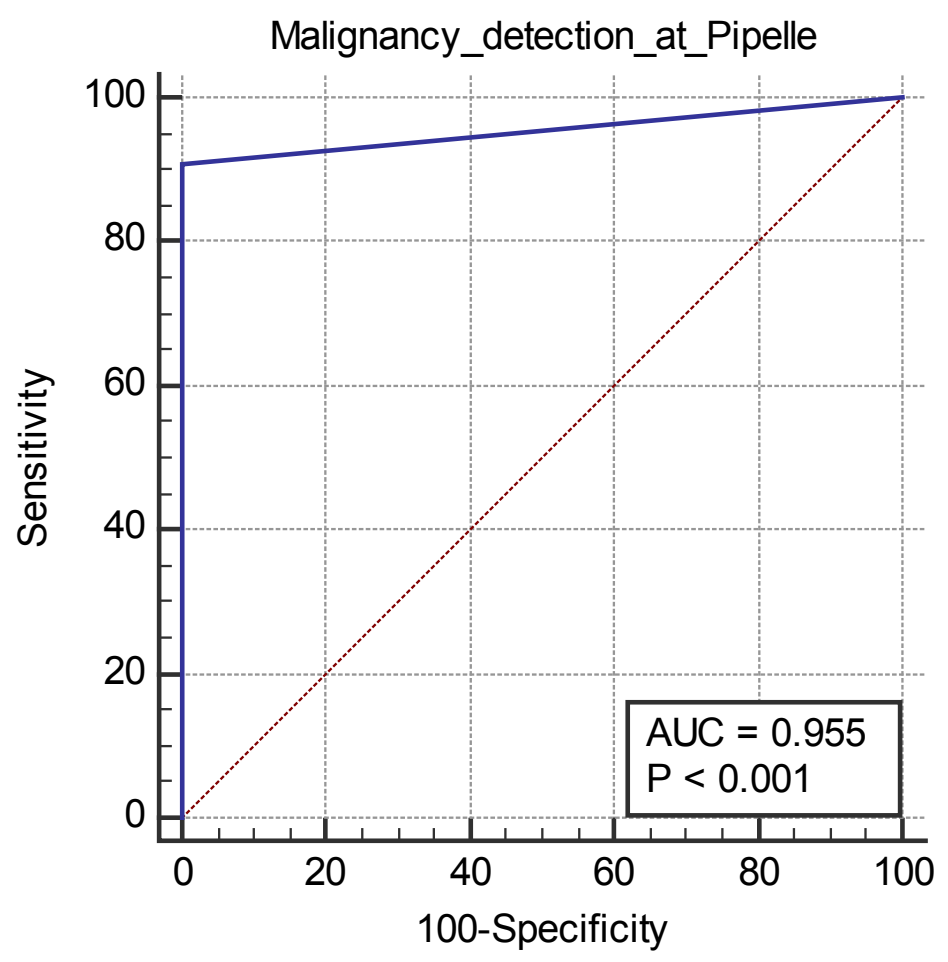

Figure 3. ROC curve of Pipelle validity.

By using ROC-curve analysis, (SHG + Pipelle) method, detected patients with malignancy, with perfect accuracy, sensitivity $=100 \%$ and specificity $=100 \%(\mathrm{p}$ $<0.01$ ) (Figures 4-9).

\section{Discussion}

In the present study, we analyzed and compared 176 cases of PMB; all patients were subjected to transvaginal ultrasonographic evaluation, saline infusion sonohysterograph and pipelle outpatient endometrial sampling.

- The most common endometrial lesion detected by hysteroscopic guide biopsy and/or hysterectomy was malignancy (50\%) followed by atrophic endometrium (22.7\%) with 16 cases of hyperplasia (9.1\%), 16 cases of endometrial polyps $(9.1 \%)$ and 16 cases of myoma $(9.1 \%)$.

- In our study each of the diagnostic tools (TVS, SHG and pipelle) were compared to hysteroscopic guided biopsy and/or hysterectomy in diagnosis of different endometrial lesions regarding Sensitivity, specificity, PPV, NPV, accuracy and the validity of each tool in detection of malignancy and also the assessment of endometrial thickness by (TVS and SHG) was calculated.

TVS has been reported to fail in diagnosis of 48 cases of suspicious mass which were misdiagnosed as hyperplasia also 8 cases of suspicious mass were misdiagnosed as endometrial polyp and 8 cases of endometrial polyp were misdiagnosed as endometrial hyperplasia while SHG failed in diagnosis of only 8 cases of suspicious mass which were misdiagnosed as endometrial polyp with respect to the pathological diagnosis. 


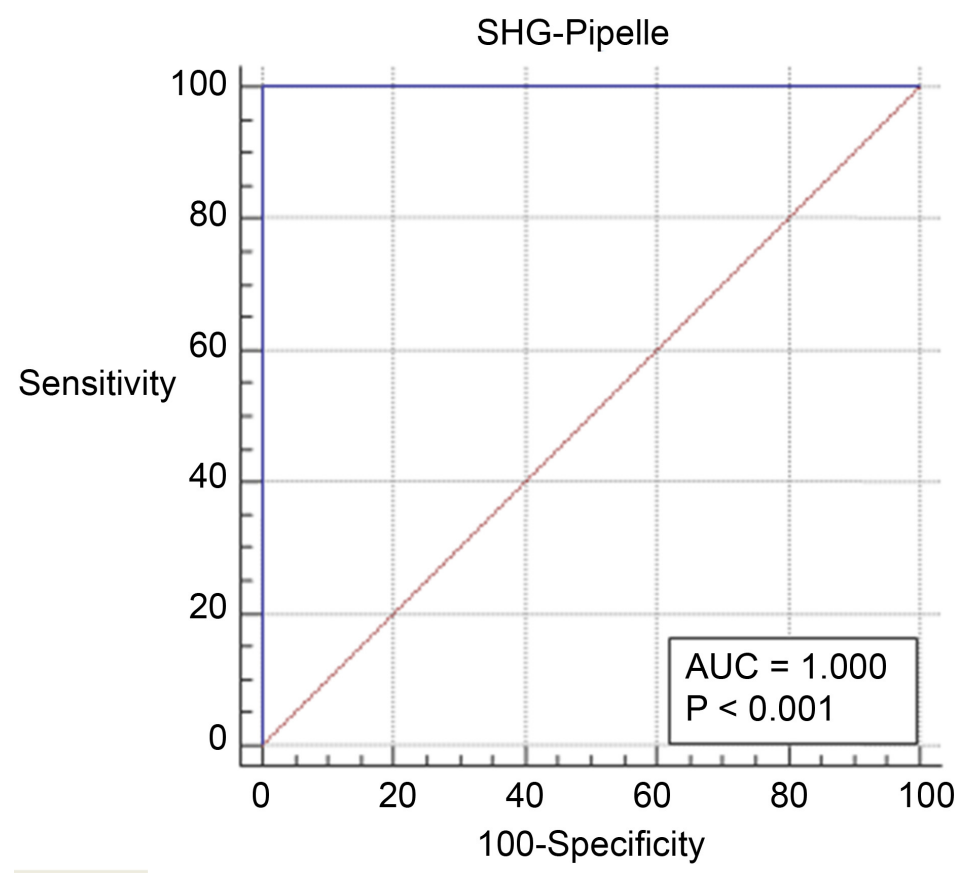

Figure 4. ROC curve of (SHG + Pipelle) validity.

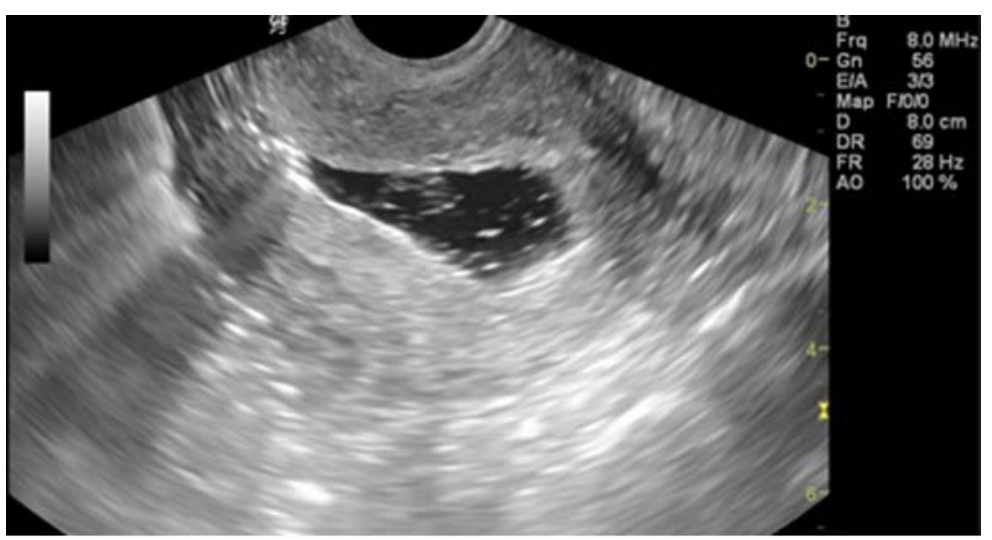

Figure 5. Thickened endometrium $(18 \mathrm{~mm})$ in menopausal female by SHG.

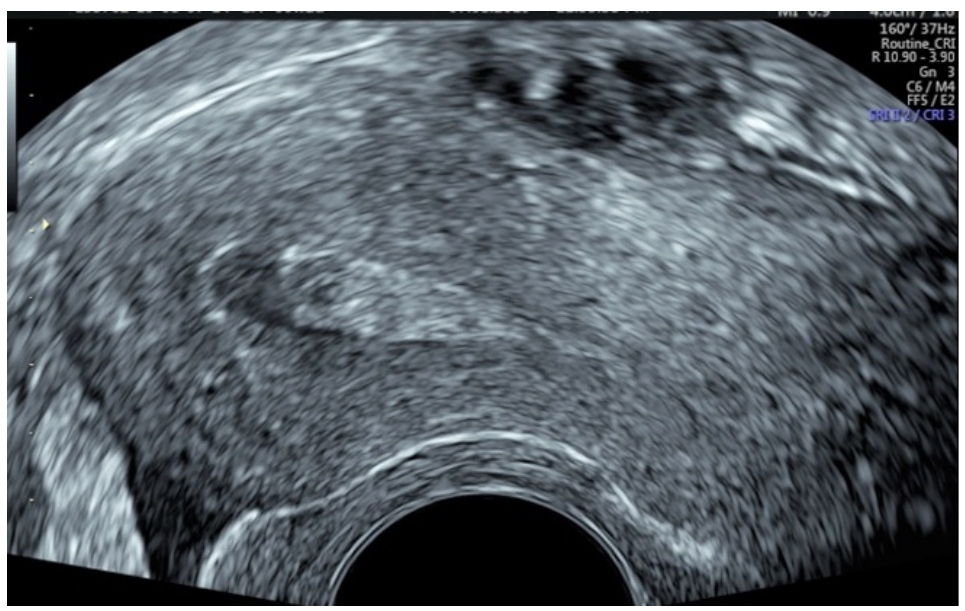

Figure 6. Thickened endometrium $(13 \mathrm{~mm})$ in menopausal female by TVS. 


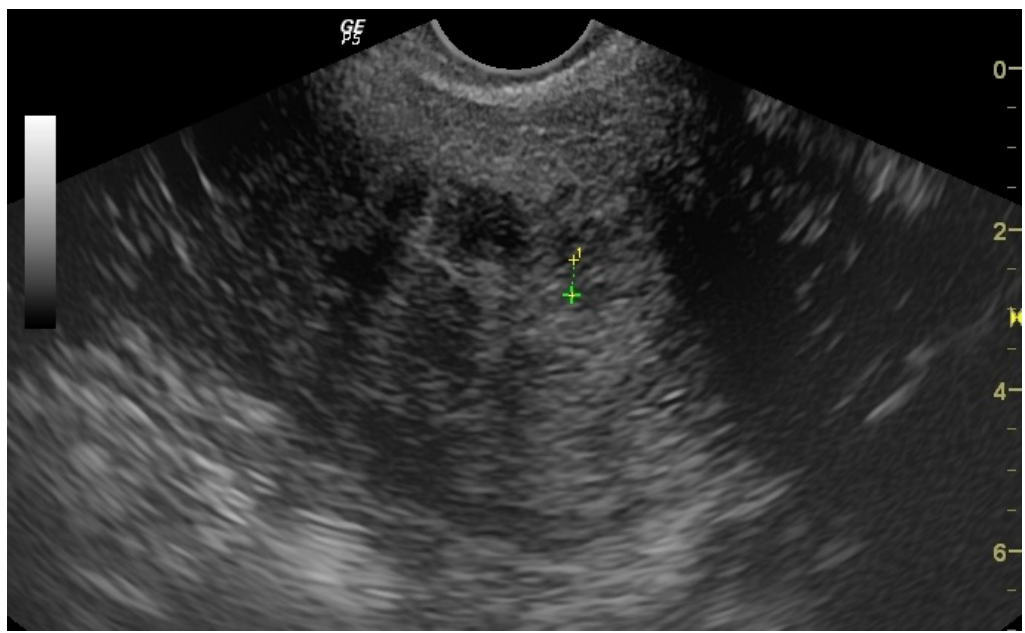

Figure 7. Atrophic endometrium (2 mm) by TVS.

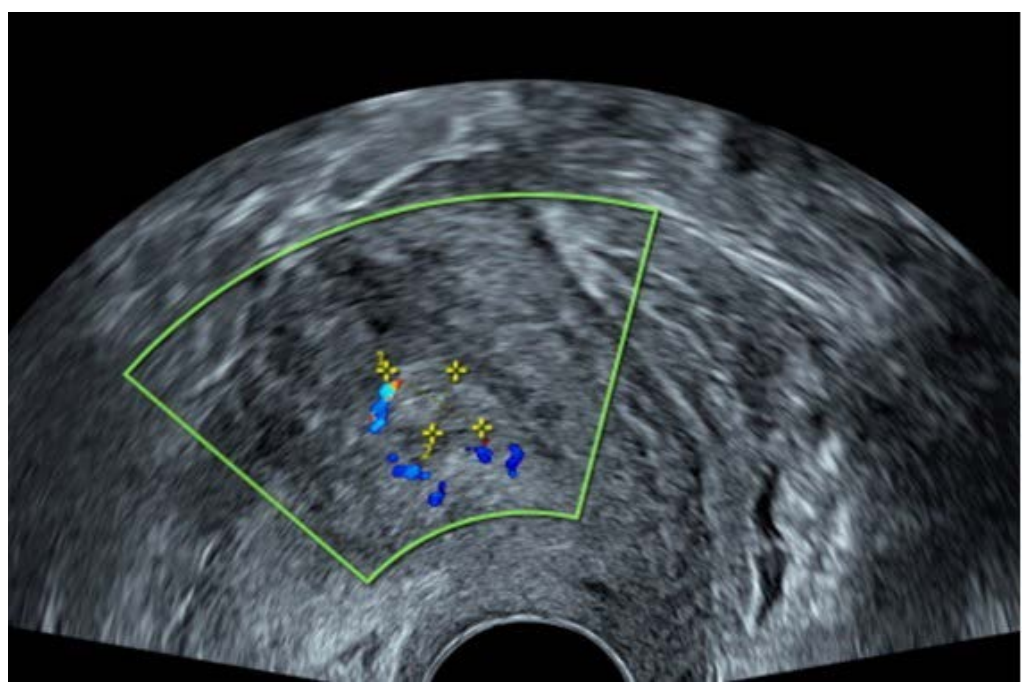

Figure 8. Hyperplasia by (14 mm) TVS.

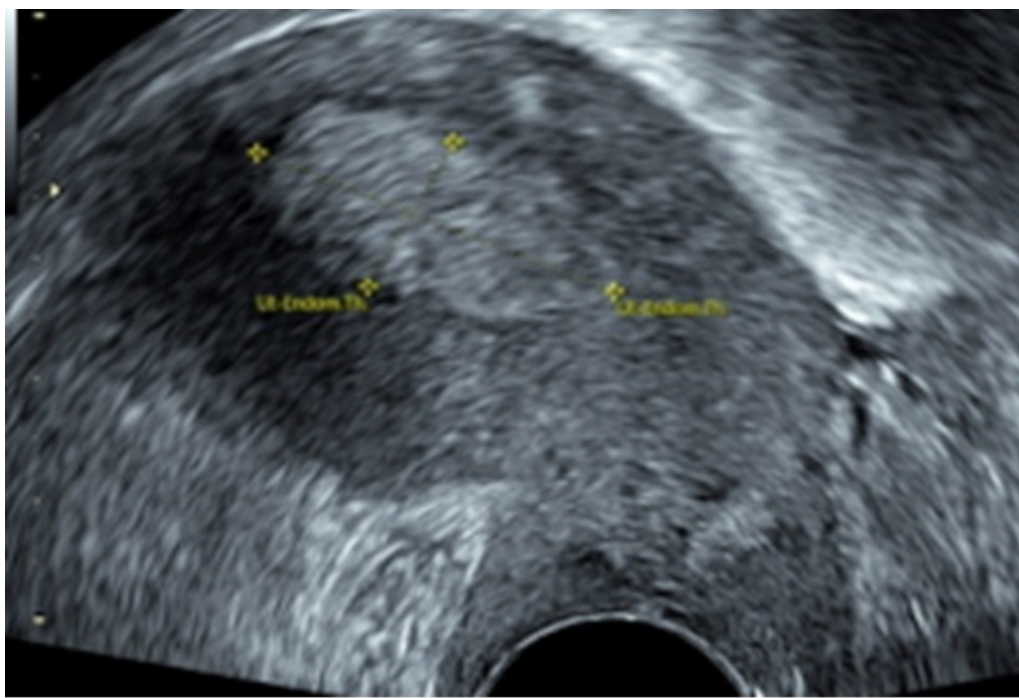

Figure 9. Hyperplasia (15 mm) by TVS. 
In cases of myoma and endometrial atrophy the diagnostic accuracy of SHG and TVS was identical (sensitivity of $100 \%$, PPV of $100 \%$ and accuracy of $100 \%$ for both).

While in cases of suspicious mass SHG is more superior than TVS (sensitivity $90.91 \%$ and $63.64 \%$, accuracy $95.45 \%$ and $81.82 \%$ respectively) with respect to histopathology.

In comparing the validity of TVS and SHG we found that highly significant decrease in malignancy assessment in the TVS method (sensitivity $=63 \%$ ), than SHG that reported non-significant difference in malignancy assessment (sensitivity $=91 \%$ and specificity $=100 \%$ ) compared to hysteroscopic-guided biopsy.

This result implied that TVS alone was not valid in malignancy detection while SHG, was close in validity to results of hysteroscopic-guided biopsy and/or hysteroscopy in malignancy detection.

This was considered to be in near alignment with the research performed by Bingol et al. (2011) in their research TVS, SIS and hysteroscopy had a sensitivity of 69.2 per cent, 92.3 per cent and 94.8 per cent; a precision of 80.6 per cent, 97.9 per cent and 98.9 per cent; a PPV of 58.7 per cent, 94.7 per cent and 97.3 per cent; and an NPV of 68.6 per cent, 96.9 per cent and 97.9 per cent respectively. For polypoid lesions, TVS, SIS and Hysteroscopy revealed a sensitivity of 55.7 percent, 96.1 percent and 98.0 percent; a specificity of 84.7 percent, 95.3 percent and 96.5 percent; PPV of 69.0 per cent, 95.3 per cent and 94.4 per cent; and finally NPV of 75.7 per cent, 95.7 per cent and 98.8 per cent respectively. The susceptibility for myomas, TVS, SIS and Hysteroscopy was 65.0 per cent, 70.0 per cent and 70.0 per cent; the accuracy was 85.0 per cent, 94.4 per cent and 94.4 per cent; the PPV was 81.2 per cent, 93.3 per cent and 93.3 per cent; and finally the NPV was 70.8 per cent, 73.9 per cent and 73.9 per cent, respectively [8] [9].

In comparison, our findings are consistent with what Mishra et al. (2015) reported in their analysis to compare the results of TVS and SIS with hysteroscopy and histopathological assessment in $42 \mathrm{PMB}$ cases. Sensitivity and specificity of TVS was $73.9 \%$ and $73.7 \%$ respectively, the positive predictive value of TVS was $77.3 \%$ and the diagnostic accuracy of TVS was $73.8 \%$ [10].

Regarding TVS invalidity in the detection of malignancy Our results agree with da Silva Wanderley et al. (2016) in their research to determine the efficacy of transvaginal ultrasonography, hysteroscopy and uterine curettage in the treatment of endometrial polyps, submucous myoma and endometrial hyperplasia, using the histopathological analysis of biopsy samples collected as a gold standard. Collected during hysteroscopy or uterine curettage and reported that although 4 histopathological cases of endometrial cancer were detected, no ultrasound evidence of malignancy was recorded in 191 tests [11].

In our study mean endometrial thickness measured by TVS was $6.2 \pm 3.4$ in benign cases and $19.2 \pm 10.8$ in malignant cases while that measured by SHG was $3.73 \pm 1.58$ in benign cases and $16.55 \pm 11.38$ in malignant cases.

No cases of endometrial cancer could be detected below cut-off endometrial thickness of $4 \mathrm{~mm}$ using SHG which is found to be more accurate in assessment 
of endometrial thickness than TVUS, when we used lower $3 \mathrm{~mm}$ as a cut-off value the sensitivity was $100 \%$ but the corresponding false positive rates were unacceptably high with $75 \%$, the best cut-off value that gives good sensitivity and also acceptable specificity was found to be $5 \mathrm{~mm}$ in SHG and $9 \mathrm{~mm}$ in TVS Using ROC curve analysis SHG reported more superior results than TVS in detection of possible malignancy at lower cut-off endometrial thickness with sensitivity $90.91 \%$ and specificity $86.37 \%$ at cut off value $>5 \mathrm{~mm}$ while TVS reported sensitivity of $72.73 \%$ and specificity $90.91 \%$ at cut off value $>9 \mathrm{~mm}$.

Wong et al. (2016) surveyed 4383 women in their cohort sample. TVS assessment of ET was collected between 2002 and 2013 in women with PMB, and the findings were diagnosed in 166 women with endometrial cancer. The mean ET was slightly higher in people with endometrial cancer than in those with stable conditions (15.7 versus $3.2 \mathrm{~mm}, \mathrm{P}<0.001$ ). The ROC curve area was 0.92 (95 per cent CI 0.89 - 0.9). At these ranges the resulting accuracy figures were 45.3 percent (95 percent CI 43.8 - 46.8 percent), 66.8 percent (65.4 - 68.2 percent), and 74.0 percent (72.7 - 75.4 percent). And concluded that using a $3 \mathrm{~mm}$ cut-off, transvaginal ultrasound is extremely able to diagnose endometrial cancer, and may identify people with $\mathrm{PMB}$ who are highly unlikely to have endometrial cancer, thus avoiding more invasive endometrial biopsy [12].

Furthermore, our results also agree with what stated by Patel et al. (2017) in their study to evaluate the clinical correlation between the TVUS endometrial thickness and histopathologic findings on endometrial biopsies in postmenopausal women with PMB. And they found that out of the 304 women in the study, $32(10.5 \%)$ had endometrial carcinoma. When examining the mean endometrial thickness 4 to $5 \mathrm{~mm}$ endometrial thickness has been used historically as a cut-off point to exclude the possibility of endometrial carcinoma. In their study, 3 out of the 32 patients with endometrial carcinoma had a TVUS-measured endometrial thickness $\leq 4 \mathrm{~mm}$. and they concluded that for a screening test, sensitivity must be high for a test to be effective as a screening tool, sensitivity is maximized at lower cut-offs and approaches $97 \%$ at a cut-off $\leq 3 \mathrm{~mm}$. The specificity, PPV, and test accuracy, however, remain very low at lower cut-offs. Analysis of o ROC found that the most ideal "cut-off" value to maximize sensitivity and specificity for TVUS is $13 \mathrm{~mm}$. At $13 \mathrm{~mm}$, sensitivity is $65.6 \%$ and specificity is $78.6 \%$. Ultimately, using the $13-\mathrm{mm}$ cut-off would lead to a delay in diagnosis in $34.4 \%$ of patients with endometrial cancer which is unacceptable. TVUS findings lacked sufficient sensitivity and specificity for the purposes of diagnosing or excluding endometrial carcinoma [13] [14].

- Regarding our study outpatient endometrial sampling "pipelle". The sensitivity, specificity, PPV, NPV, accuracy and its validity in detection of malignancy were calculated with respect to histopathology obtained by hysteroscopic guided biopsy and/or hysterectomy.

In cases of hyperplasia results was $(100 \%, 85 \%, 40 \%, 100 \%$ and $86.36 \%$ respectively).

In cases of malignancy results was $(90.91 \%, 100 \%, 100 \%, 91.67 \%$ and $95.45 \%$ 
respectively).

In atrophic endometrium results was $(60 \%, 100 \%, 100 \%, 89.47 \%$ and $90.91 \%$ respectively).

In cases of polypoid lesions and submucous myoma results were sensitivity $0 \%$, specificity $100 \%$, NPV $90.91 \%$ and accuracy $90.91 \%$.

In our study pipelle couldn't obtain an adequate sample for histopathology in $8(18.1 \%)$ cases 16 cases of them were diagnosed as atrophic endometrium, 8 cases were diagnosed as submucosal myoma and unfortunately 8 cases had a focal malignant lesion with respect to histopathology.

But fortunately according to the validity of pipelle in detection of malignant lesion it was found to detect 80 cases out of 88 cases of malignancy with sensitivity $90.9 \%$, specificity $100 \%$ and $95.45 \%$ accuracy with respect to histopathology by hysteroscopy guided biopsy and/or hysterectomy.

In this regard our results in accordance with past studies were found to agree with Lau and Spiryda (2016) in their study. In meta-analysis, the Pipelle low-pressure endometrial suction curette showed the highest sensitivity of $99.6 \%$ in the detection of endometrial cancer and $82 \%$ in the detection of low-complication endometrial hyperplasia. Endometrial biopsies are not immune to anatomical malfunctions such as endometrial polyps and leiomyomas [15] [16].

Pipelle was of greater acceptability than $\mathrm{D} \& \mathrm{C}$ although sufficient tissue for histopathological analysis was collected in slightly fewer cases than D\&C (98 vs. 100 per cent), Pipelle was approved at 98 per cent and D\&C at 34 per cent [17] [18].

The outcomes of Pipelle sampling were matched between 1996 and 1999 with other methods, such as D\&C, hysteroscopy or hysterectomy, and the conclusion was that Pipelle was the best method for detecting endometrial lesions in both postmenopausal and premenopausal women with a detection rate of $99.6 \%$ and 91\% respectively (Demirkiran et al. 2012) [19], also Gungorduk et al. (2014) [20].

- In our study we found that the validity of combination test between (SHG + Pipelle) improves the sensitivity and specificity in detection of malignancy than each method alone with perfect accuracy, sensitivity $=100 \%$ and specificity $=100 \%$ and this was found to be as an effective method in detection of malignancy.

In this regard, no past studies could be found in the literatures that combine SHG and Pipelle as a diagnostic tool in women with post menopausal bleeding and this may need further studies to answer the question of whether combination of SHG and Pipelle with their low cost, availability and easier to be learned can replace hysteroscopy in diagnosis of post menopausal bleeding or not?

\section{Conclusions}

Hysteroscopy is the gold standard for the diagnosis of cervical and uterine pa- 
thologies, and also has a therapeutic role in different benign lesions.

SHG was a reliable and accurate method in the assessment of different endometrial and uterine cavity pathologies and when combined to outpatient endometrial sampling "pipelle", the results were the same as hysteroscopy.

So it can be a good alternative to hysteroscopy. It is also cheep, available and easy to be applied in outpatient clinics especially for patients whose general anesthesia carries a great risk.

\section{Conflicts of Interest}

The authors declare no conflicts of interest regarding the publication of this paper.

\section{References}

[1] Hayatullah, G., Song, J.R. and Mirwais, A. (2018) Hysteroscopy Assessment of Endometrial Pathology with Endometrial Thickness Cut-Off Value $5 \mathrm{~mm}$ in Postmenopausal Women with Vaginal Bleeding.

[2] Lau Trevin, C., Lisa, B. and Spiryda, C. (2017) Diagnosis and Management of Postmenopausal Bleeding. In: Shoupe, D., Ed., Handbook of Gynecology, Springer, Cham, 509-515. https://doi.org/10.1007/978-3-319-17798-4_46

[3] Ghanbarzadeh, N., Haghighi, F., Semnani, F.N., Semnani, A.N. and Sa'adatpoor, E. (2015) Pathological Patterns of Endometrial Curettage Samples in Women Referred with Abnormal Uterine Bleeding: A Descriptive Study. Journal of Surgery and Trauma, 3, 39-45.

[4] Qurrat-Ul-Ain Ihsan, H.M. and Khan, K.A. (2018) Diagnostic Accuracy of Transviginal Sonography in Detecting Endometrial Hyperplasia in Post Menopausal Women. Kanem Journal of Medical Sciences, 11, 272.

[5] Grimbizis, G.F., Tsolakidis, D., Mikos, T., Anagnostou, E., Asimakopoulos, E., Stamatopoulos, P. and Tarlatzis, B.C. (2010) A Prospective Comparison of Transvaginal Ultrasound, Saline Infusion Sonohysterography, and Diagnostic Hysteroscopy in the Evaluation of Endometrial Pathology. Fertility and Sterility, 94, 2720-2725. https://doi.org/10.1016/j.fertnstert.2010.03.047

[6] Maravi, P., Verma, V., Kaushal, L., Patil, A. and Ahirwar, C. (2016) Saline Infusion Sonography in Assessment of Endometrial Pathologies in Patients with Thickened Endometrium Presenting with AUB. Journal of Evolution of Medical and Dental Sciences, 5, 2498-2502. https://doi.org/10.14260/jemds/2016/583

[7] Du, J., Li, Y., Lv, S., Wang, Q., Sun, C., Dong, X. and Batchu, N. (2016) Endometrial Sampling Devices for Early Diagnosis of Endometrial Lesions. Journal of Cancer Research and Clinical Oncology, 142, 2515-2522. https://doi.org/10.1007/s00432-016-2215-3

[8] Rezaei, M., Mohammadi, R., Raheahagh, R. and Shahoei, R. (2018) Evaluation Pathology Results in Diagnosis of Abnormal Uterine Bleeding among Women Referred to Beast Hospital of Sanandaj, 2012. Medical Journal of Tabriz University of Medical Science \& Health Service, 40, 49-54.

[9] Bingol, B., Gunenc, M.Z., Gedikbasi, A., Guner, H., Tasdemir, S. and Tiras, B. (2011) Comparison of Diagnostic Accuracy of Saline Infusion Sonohysterography, Transvaginal Sonography and Hysteroscopy in Postmenopausal Bleeding. Archives of Gynecology and Obstetrics, 284, 111-117. 
https://doi.org/10.1007/s00404-010-1604-0

[10] Mishra, R., Misra, A.P. and Mangal, Y. (2015) To Compare the Results of TVS and SIS with Hysteroscopy and Histopathological Examination in Perimenopausal \& Postmenopausal Bleeding. Journal of Evolution of Medical and Dental Sciences, 4, 1230-1237. https://doi.org/10.14260/jemds/2015/170

[11] da Silva Wanderley, M., Álvares, M.M., de Fátima Brito Vogt, M. and Sazaki, L.M.P. (2016) Accuracy of Transvaginal Ultrasonography, Hysteroscopy and Uterine $\mathrm{Cu}$ rettage in Evaluating Endometrial Pathologies. RBGO Gynecology and Obstetrics, 38, 506-511. https://doi.org/10.1055/s-0036-1593774

[12] Wong, A.W., Lao, T.H., Cheung, C.W., Yeung, S.W., Fan, H.L., Ng. P.S. and Sahota, D.S. (2016) Reappraisal of Endometrial Thickness for the Detection of Endometrial Cancer in Postmenopausal Bleeding: A Retrospective Cohort Study. BJOG: An International Journal of Obstetrics \& Gynaecology, 123, 439-446.

https://doi.org/10.1111/1471-0528.13342

[13] Schramm, A., Ebner, F., Bauer, E., Janni, W., Friebe-Hoffmann, U., Pellegrino, M. and Friedl, T.W. (2017) Value of Endometrial Thickness Assessed by Transvaginal Ultrasound for the Prediction of Endometrial Cancer in Patients with Postmenopausal Bleeding. Archives of Gynecology and Obstetrics, 296, 319-326. https://doi.org/10.1007/s00404-017-4439-0

[14] Patel, V., Wilkinson, E.J., Chamala, S., Lu, X., Castagno, J. and Rush, D. (2017) Endometrial Thickness as Measured by Transvaginal Ultrasound and the Corresponding Histopathologic Diagnosis in Women with Postmenopausal Bleeding. International Journal of Gynecological Pathology, 36, 348-355. https://doi.org/10.1097/PGP.0000000000000344

[15] Lau, T.C. and Spiryda, L.B. (2016) Diagnosis and Management of Postmenopausal Bleeding. In: Shoupe, D., Ed., Handbook of Gynecology, Springer, Cham, 1-7. https://doi.org/10.1007/978-3-319-17002-2_46-1

[16] Sanam, M. and Majid, M.M. (2015) Comparison the Diagnostic Value of Dilatation And Curettage versus Endometrial Biopsy by Pipelle-A Clinical Trial. Asian Pacific Journal of Cancer Prevention, 16, 4971-4975.

[17] Rauf, R., Shaheen, A., Sadia, S., Waqar, F., Zafar, S., Sultana, S. and Waseem, S. (2014) Outpatient Endometrial Biopsy with Pipelle versus Diagnostic Dilatation and Curettage. Journal of Ayub Medical College Abbottabad, 26, 145-148.

[18] Dijkhuizen, F.P.H.L.J., Mol, B.W.J., Brolmann, H.A.M. and Heintz, A.P.M. (2000) The Accuracy of Endometrial Sampling in the Diagnosis of Patients with Endometrial Carcinoma and Hyperplasia-A Metaanalysis. Cancer, 89, 1765-1772. https://doi.org/10.1002/1097-0142(20001015)89:8<1765::AID-CNCR17>3.0.CO;2-F

[19] Demirkiran, F., Yavuz, E., Erene,l H., Bese, T., Arvas, M. and Sanioglu, C. (2012) Which is the Best Technique for Endometrial Sampling? Aspiration (Pipelle) versus Dilatation and Curettage (D\&C). Archives of Gynecology and Obstetrics, 286, 1277-1282. https://doi.org/10.1007/s00404-012-2438-8

[20] Gungorduk, K., Asicioglu, O., Ertas, I.E., Ozdemir, I.A., Ulker, M.M., Yildirim, G., Ataser, G. and Sanci, M. (2014) Comparison of the Histopathological Diagnoses of Preoperative Dilatation and Curettage and Pipelle Biopsy. European Journal of $G y$ naecological Oncology, 35, 539-543. 